\title{
Psychometric evaluation of the Perinatal Illness Perceptions Scale (PIPS)
}

\begin{abstract}
Purpose: Perinatal mental health $(\mathrm{PMH})$, is acknowledged as a significant public health issue associated with significant personal, family, social and economic burden. Research demonstrates that healthcare practitioners lack knowledge and confidence in this area but there is likely to be a complexity of factors that may influence practitioner behaviours, including negative attitudes toward people with mental health and inaccurate illness perceptions. This study sought to evaluate the psychometric properties of the Perinatal Illness Perceptions scale (PIPS), a conceptual derivation of the Illness Perception Questionnaire -Revised

Design: A cross-sectional and exploratory instrument development design, using exploratory factor analysis was employed.

Findings: The scale demonstrated good psychometric properties revealing 3 sub-scales; Causes, Consequences (Mother); Consequences (Baby)

Originality: Our findings implicate the PIPS as the first robust psychometric measure, which can be used to in the assessment of practitioner knowledge of the causes and consequences of PMH. The PIPS could offer the opportunity to assess these domains within both educational and training context and identify practitioner attitudes which may affect clinical decision making and referral decisions.
\end{abstract}

\section{Keywords}

Perinatal; mental health; midwives; IPQ-R; psychometrics; knowledge; illness perceptions 


\section{Psychometric evaluation of the Perinatal Illness Perceptions Scale (PIPS)}

\section{Introduction}

Perinatal mental health (PMH), is a significant global public health issue (NICE 2014). This has established a clear remit for maternity practitioners in the identification and assessment of a range of PMHP problems ((PMHPs): Noonan et al, 2017; Howard et al., 2018).

The significance of PMH is not only the mental health of the mother (Kalief et al, 2015) but beyond the perinatal period on the long-term health, social, emotional, cognitive and behavioural development of children particularly among disadvantaged populations, with clear economic consequences (Bauer et al, 2014). Early intervention does have the potential to positively impact and maternal and child outcomes (Kalief et al, 2015), yet failures to appropriately identify and manage women with PMH continue (RCOG, 2017).

Midwives and health visitors (Jones et al, 2015) express concern regarding their knowledge and skills in assessing and managing women with PMHPs (Noonan et al, 2017) with knowledge deficit identified as a barrier to care (Byatt et al, 2013; Higgins et al, 2016). Beyond knowledge and confidence there is a complexity of factors that may influence practitioner behaviours, including negative attitudes toward people with mental health problems (Schafer et al, 2011). This affects understanding of PMHPs and may be linked to a lack of experience and familiarity with mental health issues but also perception of an illness. Practitioners form mental representations of an illness, which can determine how they respond emotionally to an individual with that illness. The significant issue here is that practitioners due to their own backgrounds, experiences and professional training may have differing perceptions of a condition, this can lead women down different illness trajectories and care pathways (Petrie and Weinmann, 2006), which can impact recovery and outcomes (McCorkindale et al, 2017). Knowledge, particularly when contextualised, leads to more accurate illness perceptions (Jomeen et al, 2009) but inaccurate illness perceptions may have implications for how knowledge is both interpreted and applied.

Training can improve knowledge and attitudes to PMH (Higgins et al, 2016; Jones et al, 2015; Reed et al, 2014), with associated measures developed to evaluate the constructs (Martin et al, 2017) yet much less attention has been given to intrinsic factors that influence practitioners in their decision making around PMH. Appropriate care of PMHPs requires accurate understandings of $\mathrm{PMH}$ and their 
consequences. To both understand these intrinsic factors more effectively, to inform training and to be able to evaluate the efficacy of training a valid and reliable measure is required.

\section{Measuring Illness Perceptions}

Currently the majority of studies focusing on illness perceptions use Leventhal's Self-Regulatory Model, a theoretical framework developed to explain why and how illness perceptions can differ. The identified different components includes illness identity, timeline, cause(s), consequences and control (Arat et al, 2016). This work underpinned the development of the Illness Perception Questionnaire (IPQ), which was then revised in 2002 to the IPQ-R (Moss-Morris et al, 2002). It has been widely used in many patient populations (Arat et al, 2016) and more latterly developed for healthcare professionals working with specific groups including mental health nurses working with schizophrenia (Fleming et al, 2009) learning disability nurses and schizophrenia (McCorkindale et al, 2017), nursing students and myocardial infarction (Grankvist and Brink, 2009) nurses and epileptic/nonepileptic seizures (Worsley et al, 2011) and health professionals and physical diseases (Arat et al, 2016).

\section{Measuring Illness Perceptions in PMH}

With regard to maternity, the measure has been adapted to assess midwives illness perceptions of antenatal depression (Jomeen et al, 2009) and then for health visitors and PMH more broadly (Jones et al, 2015). The adapted IPQ-R for PMH (Jones et al, 2015) generated additional items from the literature on perinatal, anxiety, depression and severe mental illness and to address overlap of somatic symptoms, physical symptoms of pregnancy were also added. Adaptations were discussed and refined by a group of practitioners with knowledge and experience of PMH from midwifery, psychiatry and clinical psychology. The brief of the expert panel was to ensure that content (i) reflected the IPQ-R domains, (ii) was specific to PMH and, (iii) easy to complete and (iv) easy to score and interpret. The adapted IPQ-R was piloted with a small group for face validity. Confirmatory factor analysis (CFA) was then used to determine model fit; essentially, how well the data fits the measurement model of relationship of individual items to sub-scales and the correlational relationship between sub-scales. Moss-Morris et al. (2002) suggested that the causes items should not be used as a scale, hence was not specifically examined in the 2009 CFA. These original CFA findings indicated that the adapted IPQ-R (PMHP) did not overall offer a good model fit; the domains of timeline (acute/chronic and cyclical); control/cure (personal and treatment); emotional representation; illness coherence were not identified (Jomeen et al, 2016). This finding is interestingly consistent with other adaptations of the IPQ-R for in a mental health context where 
model fit has been identified as poor (Fleming et al, 2009), though not with adaptations, focused on physical illness where good model fit is demonstrated (Arat et al, 2016). A conclusion of the Jomeen et al (2009) study, however, was that the consequences scale of the IPQ-R (PMHP) did show some merit and was worthy of further testing, so was used in this current study alongside the adapted IPQ-R causes items, as part of a broader questionnaire which also assessed knowledge and confidence of PMHP (Martin et al, 2017) and professional issues (Jomeen et al, 2018) related to PMH in student midwives.

The aim was to develop and evaluate key psychometric attributes including the factor structure, validity and reliability of the IPQ-R derived causes and consequences items (renamed the Perinatal Illness Perceptions Scale (PIPS).

\section{Methods}

\section{Design and participants}

A cross-sectional design and instrument evaluation approach taken to investigate the psychometric properties of the PIPS. Validity and reliability evaluation of the PIPS was undertaken using established statistical approaches (Kline, 2000; Martin and Savage-McGlynn, 2013; Martin et al, 2016).

\section{Participants}

Ten UK universities providing undergraduate midwifery programmes took part in the study. Students near completion of a BSc Midwifery, either undertaking a 3 year or 18 month programme, completed an online questionnaire. It was perceived that students late in the curriculum would have had exposure to significant theoretical and practice experience to answer the questions.

The students received study information at least a week prior to access to the questionnaire, to provide the opportunity to seek clarification. An email invitation was then sent containing a link to a Survey Monkey Questionnaire. One email reminder was sent to students. Whilst the request was to allow protected time for students to complete the questionnaire, to increase response rates, students could also complete independently.

Ethics approval was obtained from Middlesex University ethics committee (HEESC APPLICATION NUMBER: MW13) and at all study sites. Consent was embedded the beginning of the questionnaire and 266 questionnaires were fully completed. 


\section{Measures}

Perinatal IIIness Perceptions Scale (PIPS)

As described earlier the two IPQ-R sub-scale domains which appear to offer utility within the PMH field are the consequences sub-scale and the causes sub-scale, which were adapted for the purposes of this study. Higher scores on the consequences sub-scale indicate comparatively greater belief in the negative consequences associated with the presentation. Moss-Morris et al (2002) suggested that the causes items should be subject to techniques such as factor analysis to identify as a scale or sub-scale specific to a presentation. The items comprising the PIPS were scored on a 0-3 Likert scale with higher scores indicating greater agreement.

\section{Multi-dimensional Health Locus of Control (MHLC) Scale}

Locus of control was determined by adapting the illness-specific (Form C) version of the Multidimensional Health Locus of Control (MHLC) scale (Wallston et al, 1994). Item content was orientated to the perinatal period and the context of $\mathrm{PMH}$. This adaptation assesses four domains of locus of control (LC), these being Internal (6-items), Chance (6-items), Doctors (3-items) and Other people (3-items) consistent with the original versions. Higher scores indicate greater levels of the particular LC attribute. A four-point Likert format on a 0-3 rating was utilised.

\section{Single-item measures}

A single item question, 'It is easy for me to obtain help for women with mental health problems' was used to categorise participants dichotomously (YES/NO). Categorisation based on this 'help' question was used as the between-groups (YES/NO) variable to facilitate known-groups discriminant validity evaluation by examining differences between PIPS scores. A second single-item question, 'How satisfied are you with the training you have received in your degree programme on perinatal mental health?' scored on a four-point 'very unsatisfied' to 'very satisfied' Likert scaling was used to evaluate divergent validity of the PIPS, the spread of data across the four-point scale allowing any association between this index and PIPS scores to be statistically evaluated.

\section{Statistical analysis}

\section{Exploratory factor analysis}

Exploratory factor analysis (EFA) was used to determine the factor structure of the PIPS scale. The inclusion of just two IPQ-R derived sub-scales in the PIPS, the significant extent of item revision for the PIPS and the original suggestion of Moss-Morris et al (2002) that the causes items should not be 
used as a scale or sub-scale until its factor structure is known indicates a clear preference of EFA over CFA for determining factorial structure. Maximum-likelihoods (ML) estimation was used for initial factor condensation followed by oblimin rotation of extracted factors (Costello and Osborne, 2005). PIPS item univariate distributional characteristics were evaluated to identify any potential issues of non-normality since ML estimation assumes a normal distribution. Item skew values $>3$ and kurtosis values $>10$ would indicate non-normality (Kline, 2005). Similarly, multivariate outliers were detected by estimating individual participant data Mahalanobis distances (Mahalanobis, 1936; De Maesschalck et al, 2000) based on the study measures used. Recognising the ambiguity inherent to traditional methods of identifying the optimal number of factors within a dataset such as the Kaiser criterion or scree plot (Klein, 2000), a parallel analysis (Horn, 1965) was conducted to estimate with precision the number of underlying factors, this approach being supported further by comparison with Velicer's (1976) minimum average partial (MAP) criterion and the Baysian Information Criterion (BIC). Agreement between these approaches would furnish the factor number specification for conducting the EFA. A plausible item-factor loading was determined by a coefficient level of $>0.30$ to ensure optimum identification of items which would contribute usefully to subscales anticipated to be inherent to the PIPS. The EFA was evaluated using multiple fit indices (Bentler and Bonett, 1980), specifically the comparative fit index (CFI) (Bentler, 1990), the Root Mean Squared Error of Approximation (RMSEA) and the standardised root mean square residual (SRMR). CFI values greater than 0.90 indicate an acceptable model fit (Hu and Bentler, 1995) and CFI values of 0.95 or greater indicate good model fit (Hu and Bentler, 1999). RMSEA values of less than 0.05 indicate a good fit to the data and values of 0.08 or less indicate acceptable fit (Schumaker and Lomax, 2010). SRMR values of less than 0.08 indicate acceptable model fit (Hu and Bentler, 1999) while SRMR values of 0.05 or less are indicative of good model fit (Byrne, 2010).

\section{Divergent validity}

Divergent validity was determined by correlating PIPS total and sub-scale scored with the single-item 'satisfaction with PMH training' score. No significant relationship was predicted between PIPS subscales and the satisfaction score. The choice of 'satisfaction with PMH training' score was chosen specifically for divergent validity evaluation since it is important to differentiate the PIPS as a measure of perceptions of PMH rather than an entirely knowledge-based construct. Accrual of knowledge within educational and training programmes is influenced by satisfaction with educational delivery, thus the use of this question for divergent validity evaluation is important to ensure there is no confounding effect of the experience of training on $\mathrm{PMH}$ with perceptions of $\mathrm{PMH}$. 


\section{Convergent validity}

Convergent validity was evaluated by correlating PIPS sub-scale scores with specific MHLC sub-scales. It is predicted that the PIPS causes sub-scale/s scores would be significantly and negatively correlated with the 'internal' MHLC sub-scale, thus evaluating the anticipated relationship that the perception of a woman being in control of her PMH will be associated with lower extraneous causation of PMH issues. It was further predicted that PIPS consequences sub-scale/s scores would be significantly and positively correlated with the 'doctors' MHLC sub-scale, this evaluating the anticipated relationship that higher negative consequences of PMH problems are associated with a greater belief of medical intervention/control of $\mathrm{PMH}$ problems.

\section{Known-groups discriminant validity and sub-scale discriminability}

Known-groups discriminant validity was evaluated by comparing those categorised dichotomously on the 'help' question. It was predicted that those categorised as finding appropriate help easy for women with PMH problems would have statistically significant higher scores on the PIPS causes subscale/s compared to the not finding help easy group, this anticipated difference is predicated on the basis that identification of PMH causation is critical to solution-focused intervention, for example, appropriate referral pathways. It was further predicted that there would be no significant differences between these groups on PIPS consequences sub-scale scores, predicated on the basis that irrespective of perceptions of being able to find help or not, $\mathrm{PMH}$ problems are anticipated to be perceived as negative. The between-subject $t$-test was used to evaluate any group differences on PIPS sub-scale scores.

\section{Internal consistency}

Cronbach's alpha was used to evaluate the internal consistency of each PIPS sub-scales. A

Cronbach's alpha of 0.70 or greater is indicative of acceptable internal reliability (Kline, 1993; 2000).

Statistical analysis was conducted using the statistical software package R (R core team, 2013).

\section{Descriptive results}

Of 266 participants, the majority were from direct entry programmes $(\mathrm{N}=237)$ and the remainder from the 18 month shortened programme $(\mathrm{N}=29)$. The smallest number recruited from a single site was $\mathrm{N}=14$ and the largest $\mathrm{N}=44$. The majority of participants $(\mathrm{N}=191)$ were aged 30 or younger. All participants were female. Evaluation of Mahalanobis distances revealed the presence of 18 multivariate outliers in the dataset and these participants were consequently excluded from further analysis (final dataset $\mathrm{N}=248$, direct entry $\mathrm{N}=221(89 \%)$, conversion course $\mathrm{N}=27(11 \%)$ ). The means, 
standard deviations, skew and kurtosis of each PIPS item are shown in Table 1. below. Skew and kurtosis characteristics for each item indicate a univariate normal distribution (skew $<3$, kurtosis $<10)$.

\section{TABLE 1. ABOUT HERE}

\section{Exploratory factor analysis}

The Kaiser-Meyer-Olkin measure of sampling adequacy (0.88) and the Bartlett test of sphericity $\left(\chi^{2}=2489.76, d f=325, p<0.001\right)$ indicated suitability for EFA. Parallel analysis suggested three factors to be optimum, an observation supported by both the Velicer MAP which achieved a minimum of 0.01 with three factors and the empirical BIC which achieved a minimum of -840.12 with three factors. Three factors with Eigenvalues greater than 1. $(7.97,2.13 \& 1.71)$ accounted for $45 \%$ of the variance. Item-factor loadings are summarised in Table 2. Factor 1. comprised exclusively all 17 items related to the PIPS causes domain (PIPS-CAUSES). Factor 2. comprised 4 consequences items (items 1, 2, 7 \& 8) the content of which emphasised the impact of PMH problems on the woman (PIPS-MOTHER). Factor 3. comprised three items (items 4, 5 \& 9) which contextually focused on $\mathrm{PMH}$ problems in terms of consequences to the baby/infant (PIPS-BABY). The fit to data of the three-factor model was $\left(\chi^{2}(\mathrm{df}=250)=537.34, p<0.01, \mathrm{CFI}=0.87, \mathrm{RMSEA}=0.07(0.06-0.08,95 \% \mathrm{Cl})\right.$, $R M S R=0.05$, df-corrected RMSR=0.06). Two PIPS consequences items (items $3 \& 6$ ) did not load on any of the three factors and these were rejected from the PIPS and all subsequent analysis. Subscales derived from the EFA were all found to be significantly and positively correlated, PIPS-CAUSES with PIPS-MOTHER, $r=0.52, p<0.001$; PIPS-CAUSES with PIPS-BABY, $r=0.14, p=0.03$, and PIPSMOTHER with PIPS-BABY, $r=0.16, p<0.01$.

\section{TABLE 2. ABOUT HERE}

\section{Divergent validity}

No significant correlation was observed between the PIPS-CAUSES $(r=-0.002, p=0.97)$, PIPSMOTHER $(r=0.05, p=0.41)$ and PIPS-BABY $(r<-0.001, p=0.99)$ sub-scales and the single item satisfaction with PMH training question.

\section{Convergent validity}

Significant negative correlations were observed between the PIPS-CAUSES sub-scale and the MHLC Internal $(r=-0.19, p=0.002)$. The PIPS-MOTHER sub-scale was observed to be significantly and 
positively correlated with the MHLC Doctors sub-scale $(r=0.24, p<0.001)$. The PIPS-BABY sub-scale was also significantly correlated with the MHLC Doctors sub-scale $(r=0.14, p=0.03)$. The data characteristics of all MHLC sub-scales and the satisfaction with PMH question are summarised in Table 3.

TABLE 3. ABOUT HERE

\section{Known-groups discriminant validity and sub-scale discriminability}

Dichotomous categorisation of participants on the 'help' question revealed the majority to endorse the ease of finding help for women experiencing $\mathrm{PMH}$ problems response $(\mathrm{N}=145,58 \%)$. Independent $t$-tests revealed statistically significant differences between groups on the PIPS-CAUSES sub-scales in the direction predicted (Figure 1.). No statistically significant differences were observed between groups on the PIPS-MOTHER and PIPS-BABY sub-scale scores. A summary of between-groups data characteristics, analyses and effect size estimations are shown in Table 4.

\section{FIGURE 1. ABOUT HERE}

TABLE 4. ABOUT HERE

\section{Internal consistency}

Calculated Cronbach's alpha of the PIPS-CAUSES, PIPS-MOTHER and PIPS-BABY sub-scales were 0.90 $(0.89-0.92), 0.75(0.70-0.80)$ and $0.63(0.54-0.71)$ respectively $(95 \%$ confidence intervals in parentheses).

\section{Discussion}

The current study sought to determine the psychometric robustness and applied utility of the IPQ-R (Moss-Morris et al, 2002) 'causes' and 'consequences' derived domains, developed for PMH. Noteworthy prior to discussion of the empirical findings is that the focus on these particular domains was informed by work from a previous study (Jomeen et al, 2014); as per Fleming et al (2009) and Martin et al (2016) application of the IPQ-R sub-scales to mental health contexts is seemingly problematic in terms of measurement veracity, in a way which it is not in a physical health context (Arat et al, 2016) which may be fundamental to the underlying dimensional structure as envisaged by Moss-Morris et al (2002). 
The EFA revealed the PIPS to comprise three factors, which were unambiguously differentiated with no evidence of cross-loading items. All 'causes' items loaded on factor 1, offering evidence for the unidimensionality of this PIPS-CAUSES sub-scale and satisfying Moss-Morris et al.'s (2002) recommendation that use of the causes items should be informed by a factor analysis. Factor 2 and factor 3 exclusively represented items from the 'consequences' sub-scale and the differentiating feature of item content was whether the consequences were attributable to the mother (factor 2. , four-items, PIPS-MOTHER sub-scale) or the baby/infant (factor 3., three-items, PIPS-BABY sub-scale). This differentiation of the consequences items is potentially useful in determining the perspectives of practitioners on PMH issues. Given that most of the economic cost is related to child outcomes (Bauer et al, 2014), the utility of the PIPS-BABY sub-scale in highlighting and assessing this dimension of knowledge, in relation to PMH outcomes, seems of merit within the context of training and education. A further observation is that the content of the PIPS-BABY sub-scale has items, which relate to both baby and infant and thus highlights continuity in the trajectory of PMH outcomes. These are not necessarily within the traditional boundaries of education for midwives but are critical nonetheless to midwives knowledge of the enduring nature and burden of PMHP, if not effectively identified and managed. The PIPS-MOTHER sub-scale, in terms of item content also clearly demonstrates a continuity of the impact of PMH issues beyond that often circumscribed by midwifery curriculums. This may also have relevance for perinatal practitioners outside midwifery, but also to those practitioners whose encounters with children with mental health problems, which may be consequential of PMHP.

Taken together, the PIPS-MOTHER and PIPS-BABY sub-scales would appear to have good face validity and utility. Appraisal of the validity and reliability testing also largely supports, the potential utility and applicability of these two PIPS sub-scales. The highly significant correlation between the PIPSCAUSES sub-scale and the PIPS-MOTHER sub-scale highlights the conceptual relatedness between these sub-scale domains, essentially that practitioners clearly identify 'cause and effect' relationships to the development and occurrence of $\mathrm{PMH}$ problems. Interestingly, this relationship would appear to be less conceptually-related to the development of associated problems within the baby/infant, evidenced by the lower degree of association (though still statistically significant) between the PIPS-CAUSES and PIPS-BABY sub-scales. This observation may represent a 'disconnect', as alluded to above, created by professional care boundaries and perceived responsibilities, which in midwifery would be perceived to end within the first 28 days. It may also reflect a failure to identify PMH as an intrinsic component of well-being through the life course, which has implications for both undergraduate curriculum and post-registration training content. 
It was noted that both the PIPS-WOMAN and PIPS-BABY sub-scales demonstrated adequate divergent and convergent validity characteristics. The internal consistency characteristics of the PIPS-WOMAN sub-scale was observed to be acceptable by conventional criteria (Kline, 2000) though the PIPS-BABY sub-scale was sub-optimal (0.63). Authors have suggested that a Cronbach's alpha of 0.60 and above may be acceptable in certain circumstance (Nunnally, 1967), particularly in exploratory studies (Hair et al, 2006). Given that alpha is also influenced by the number of items comprising a scale, and that the PIPS-BABY sub-scale was modest $(N=3)$, further research is required on the sub-scale to confirm its internal reliability characteristics and potentially revise its content, by the addition of expert or empirically generated items.

The PIPS-CAUSES sub-scale, as observed from the EFA findings, represents a uni-dimensional construct of clear relevance and importance to clinical training and education. The EFA offers clear support for the use of the 'causes' items as a defined sub-scale and this is supported by the validity and reliability testing conducted. It was observed that the known-groups validity testing clearly differentiated groups categorised by ease of finding help for women with $\mathrm{PMH}$ problems. This observation is consistent with the theoretical tenet that practitioners with a greater awareness of the causes of PMH problems during the course of training, would, as a consequence, be more likely to know or seek out the appropriate referral routes and contacts when encountering a mother experiencing PMH concerns. The finding also that the PIPS-CAUSES sub-scale demonstrated excellent internal consistency offers further confidence in the measurement veracity of this subscale measure.

The divergent validity observations confirm evidence of this domain of validity testing with no significant relationship observed between any of the PIPS sub-scales and student satisfaction with $\mathrm{PMH}$ training, thus indicating that the PIPS is assessing perceptions of PMH as distinct from knowledge of $\mathrm{PMH}$. It was also clear from the sub-scale discriminability analysis that the sub-scale domains represented by the PIPS-CAUSES sub-scale and both PIPS consequences sub-scales were sensitive to distinct aspects of PMH perceptions of participants as evidenced by the results of the between-subjects $t$-tests which revealed no significant differences between groups differentiated by the 'help' question on either the PIPS-MOTHER or PIPS-BABY sub-scale but a statistically significant difference as reported earlier on the PIPS-CAUSES sub-scale.

Additionally, the convergent validity findings would also give psychometric support for the usefulness of this sub-scale, given the significant negative relationship with 'internal' MHLC. Whilst 
the absolute size of the correlation was small, though significant, it is recognised that 'internal' MHLC would be anticipated to be related to the PIPS-CAUSES domain but not a proxy for it, therefore, absolute $r$ values would justifiably not be anticipated to be high. The findings related to MHLC are worthy of further interpretation. The negative internal LC correlation here implies that student midwives do not feel that women have any personal control over the causes of PMH; when combined with the finding that the PIPS-MOTHER and PIPS-BABY sub-scales are positively correlated with the MHLC Doctors sub-scale (indicating a belief that health events are most influenced/controlled by doctors) an interesting perspective emerges. One which seemingly continues to ascribe a biomedical rather than psychological (Brubaker and Dillaway, 2009) or resilience model to mitigating the consequences of $\mathrm{PMH}$. This has potentially interesting connotations for clinical decision-making and referral. Using such a framework of understanding may fail to consider the utility of psychological interventions and/or a resiliency based framework which predominantly focuses on modifying internal assets, such as coping skills and self-efficacy (Reed et al, 2014).

The study had a number of limitations, consistent with the early and exploratory context of this IPQR-derived measure. Firstly, it was noted that though the EFA produced a clear differentiation of factors consistent with clearly delineated sub-scales attributable to the established causes and consequence domains of the IPQ-R sub-scale, it was also observed that the model 'fit', though acceptable by the conventions specific to RMSEA and SRMR which indicated a 'good to excellent fit' to data, was inconsistent with the CFI findings which indicated a sub-optimal data fit $(<0.90)$. It should be noted however, that fit evaluation is based on an account on the balance of evidence from a range of fit statistics and also, that fit indices such as those used in the current study are more usually associated with confirmatory factor analysis and structural equation modelling in contrast to EFA. The survey approach did not allow for test-retest reliability, which can provide a further valuable index of psychometric integrity, this can be addressed in future studies.

\section{Conclusion}

The PIPS appears to provide a sound psychometric instrument for assessing student midwives knowledge of the causes and consequences of PMH. It offer the opportunity to assess these domains within both educational and training context and identify practitioner attitudes which may affect clinical decision making and referral decisions. It is easy to see how the use of the PIPS could be extended or used beyond a midwifery context for use with other professionals caring for perinatal women. The PIPS could be part of a toolkit of assessment measures that support practitioners to 
confidently and proactively identify and assess women's PMHP and deliver improved outcomes for mothers, their children and the wider family. 


\section{References}

Arat, S., Van den Zegal, A., Van Rillaer, M., Moons, P., Vandenberghe, J., De Langhe, E. and Westhovens,R. (2016). Development and preliminary evaluation of the validity and reliability of a revised illness perception questionnaire for healthcare professionals. BMC Nursing, 15(34). DOI:10.1186/s12912-016-0156-4

Bauer, A., Parsonage, M., Knapp, M., Lemmi, V. and Adelaja, B. (2014). Costs of perinatal mental problems. London: London School of Economics and Political Science.

Bentler, P. M. (1990). Comparative fit indexes in structural models. Psychol Bull, 107(2), 238-246. Bentler, P. M., \& Bonett, D. G. (1980). Significance tests and goodness of fit in the evaluation of covariance structures. Psychological Bulletin, 88, 588-606.

Brubaker, S. J. and Dillaway, H. E. (2009). Medicalization, Natural Childbirth and Birthing Experiences. Sociology Compass, 3(1), 31-48. doi:10.1111/j.1751-9020.2008.00183.x

Byatt, N., Biebel, K., Friedman, L., Debordes-Jackson, G., Ziedonis, D. and Pbert, L. (2013). Patient's views on depression care in obstetric settings: how do they compare to the views of perinatal health care professionals? General Hospital Psychiatry, 35(6), 598-604.

DOI:http://dx.doi.org/10.1016/j.genhosppsych.2013.07.011

Byrne, B. M. (2010). Structural Equation Modeling with AMOS: Basic Concepts, Applications and Programming (2nd ed.). New York: Routledge/Taylor and Francis Group.

Carlson, K. D. and Herdman, A. O. (2012). Understanding the impact of convergent validity on Research results. Organizational Research Methods, 15(1), 17-32.

Costello, A. B. and Osborne, J. (2005). Best practices in exploratory factor analysis: Four recommendations for getting the most from your analysis. Practical Assessment Research and Evaluation, 10(7).

Cronbach, L. J. (1951). Coefficient alpha and the internal structure of tests. Psychometrika, 16(3) 297-334.

De Maesschalck, R., Jouan-Rimbaud, D. and Massart, D. L. (2000). The Mahalanobis distance. Chemometrics and Intelligent Laboratory Systems, 50(1), 1-18.

de Winter, J. C., Dodou, D. and Wieringa, P. A. (2009). Exploratory Factor Analysis With Small Sample Sizes. Multivariate Behavioural Research, 44(2), 147-181. doi:10.1080/00273170902794206 Fleming, M. P., Martin, C. R., Miles, J. and Atkinson, J. (2009). The utility of the Illness Perception Questionnaire in the evaluation of mental health practitioners' perspectives on patients with Schizophrenia. Journal of Evaluation in Clinical Practice, 15(5), 826-831. doi:10.1111/j.13652753.2008.01103.x

Graham, J. M. (2006). Congeneric and (essentially) tau-equivalent estimates of score reliability: What 
they are and how to use them. Educational and Psychological Measurement, 66, 930-944.

Hair, J. F., Black, W. C., Babin, B. J., Anderson, R. E. and Tatham, R. L. (2006). Multivariate Data Analysis. Upper Saddle River, New Jersey: Pearson Prentice Hall.

Higgins, A., Tuohy, T., Murphy, R. and Begley, C. (2016b). Mothers with mental health problems: Contrasting experiences of support within maternity services in the Republic of Ireland. Midwifery, 6, 28-34. DOI: http://dx.doi.org/10.1016/j.midw.2016.02.023

Higgins, A., Carroll, M. and Sharek, D. (2016). Impact of perinatal mental health education on student midwives' knowledge, skills and attitudes: A pre/post evaluation of a module of study. Nurse Education Today, 36, 364-369. doi:10.1016/j.nedt.2015.09.007

Horn, J. L. (1965). A rationale and test for the number of factors in factor analysis. Psychometrika, 30, $179-185$

Howard, L,M., Ryan, E,G., Trevillion, K., Anderson, F., Bick, D., Bye, A., Byford, S., O’Connor, S., Sands, P., Demilew, J., Milgrom, J. and Pickles, A., 2018. Accuracy of the Whooley questions and the Edinburgh Postnatal Depression Scale in identifying depression and other mental disorders in early pregnancy. The British Journal of Psychiatry, 212, 50-56.

Hu, L. T. and Bentler, P. M. (1995). Evaluating model fit. In R. H. Hoyle (Ed.), Structural Equation Modelling: Concepts, Issues and Applications. Thousand Oaks, CA: Sage.

Hu, L. T. and Bentler, P. M. (1999). Cutoff criteria for fit indexes in covariance structure analysis: Conventional criteria versus new alternatives. Structural Equation Modeling, 6, 1-55. Jomeen, J., Glover, L. F. and Davies, S.A. (2009). Midwives' illness perceptions of antenatal depression. British Journal of Midwifery, 17(5), 296-303. Retrieved from http://search.ebscohost.com/login.aspx?direct=true\&db=aph\&AN=39664547\&site=ehost-live Jomeen, J., Glover, L., Jones, C., Garg, D., Marshall, C. and Martin, C.R. (2016). Adapting the Illness Perception Questionnaire - Revised (IPQ-R) to assess practitioner perceptions of perinatal mental health problems (PMHP) [Abstract]. Journal of Reproductive and Infant Psychology, 32:3, e1-e33, DOI: $\underline{10.1080 / 02646838.2015 .1133158}$

Jomeen, J, Jarrett P and Martin C (2018). Professional issues in maternal mental health scale (PIMMHS): The development and initial validation of a brief and valid measure. European Journal of Midwifery. 2(2). DOI: 10.18332/ejm/83276

Jones, C., Jomeen, J., Glover, L., Garg, D. and Marshall, C. (2015). Exploring changes in health visitors' knowledge, confidence and decision making for women with perinatal mental health difficulties following a brief training package. European Journal of Person Centred Healthcare 3(3), 384-391. 
Khalifeh, H., Brauer, R., Toulmin, H., Howard, L.M., 2015. Perinatal mental health: What every neonatologist should know. Early Human Development. 91(2015), 649-653.

http://dx.doi.org/10.1016/i.earlhumdev.2015.08.010

Kline, P. (1993). The Handbook of Psychological Testing. London: Routledge.

Kline, P. (2000). A Psychometrics Primer. London: Free Association Books.

Kline, R. B. (2005). Principles and Practice of Structural Equation Modeling (2nd ed.). New York: Guilford Press.

Mahalanobis, P. C. (1936). On the generalised distance in statistics. Proceedings of the National Institute of Sciences of India, 2(1), 49-55.

Martin, C. R., Fleming, M. P. and McCorkindale, S. (2016). Measurement properties of the Illness

Perception Questionnaire in practitioners working with patients with co-morbid schizophrenia and learning disability. European Journal of Person Centered Healthcare, 4(1).

DOI:10.5750/ejpch.v4i1.1073

Martin C.R., Jomeen J. and Jarrett, P. (2017). The Development and Initial Validation of the Perinatal Mental Health Awareness Scale in Student Midwives. Journal of Midwifery and Reproductive Health.

5: 1-11. DOI: 10.22038/JMRH.2017.9251

Martin, C. R. and Savage-McGlynn, E. (2013). A 'good practice' guide for the reporting of design and analysis for psychometric evaluation. Journal of Reproductive and Infant Psychology, 31(5), 449-455. doi:10.1080/02646838.2013.835036

McCorkindale, S., Fleming, M. and Martin, C.R., (2017). Perceptions of learning disability burses and support staff toward people with a diagnosis of schizophrenia. Journal of Psychiatric and Mental Health Nursing. 24, 282-292

Moss-Morris, R., Weinman, J., Petrie, K. J., Horne, R., Cameron, L. D. and Buick, D. (2002). The Revised illness perception questionnaire (IPQ-R). Psychology and Health, 17(1), 1-16.

National Institute for Clinical Excellence. (2014). Antenatal and postnatal mental health:

Clinical management and service guidance. London: Department of Health.

Noonan, M., Doody, O., Jomeen, .J and Galvin, R. (2017). Midwives' perceptions and experiences of caring for women who experience perinatal mental health problems: An integrative review.

Midwifery, 45, 56-71. DOI: https://doi.org/10.1016/j.midw.2016.12.010

Nunnally, J. C. (1967). Psychometric Theory. New York: McGraw-Hill.

Petrie, K. J. and Weinman, J. (2006). Why illness perceptions matter. Clinical Medicine (Lond), 6(6), 536-539.

Preacher, K. J. and Coffman, D. L. (2006). Computing power and minimum sample size for RMSEA [Computer software]. 
RCOG. (2017). Maternal mental health - women's voices. Retrieved from London:

https://www.rcog.org.uk/globalassets/documents/patients/information/maternalmentalhealthwom ensvoicespdf

Reed, M., Fenwick, J., Hauck, Y., Gamble, J. and Creedy, D. K. (2014). Australian midwives' experience Of delivering a counselling intervention for women reporting a traumatic birth. Midwifery, 30(2), 269-275. DOI:10.1016/j.midw.2013.07.009

Revelle, W. and Rocklin, T. (1979). Very Simple Structure: An Alternative Procedure for Estimating The Optimal Number of Interpretable Factors. Multivariate Behavioural Research, 14(4), 403-414. DOI:10.1207/s15327906mbr1404_2

Revelle, W., \& Rocklin, T. (1979). Very Simple Structure: An Alternative Procedure For Estimating The Optimal Number of Interpretable Factors. Multivariate Behavioural Research, 14(4), 403-414.

DOI:10.1207/s15327906mbr1404_2

Schafer, T., Wood, S., \& Williams, R. (2011). A survey into student nurses' attitudes towards mental illness: Implications for nurse training. Nurse Education Today, 31(4), 328-332.

DOI:https://doi.org/10.1016/j.nedt.2010.06.010

Schumacker, R. E., \& Lomax, R. G. (2010). A Beginner's Guide to Structural Equation Modelling (3 ${ }^{\text {rd }}$ ed.). New York: Routledge/Taylor and Francis Group.

Team, R. C. (2013). R: A Language and Environment for Statistical Computing. Vienna, Austria: Foundation for Statistical Computing.

Velicer, W. (1976). Determining the number of components from the matrix of partial correlations. Psychometrika, 41(3), 321-327.

Wallston, K. A., Stein, M. J., \& Smith, C. A. (1994). Form C of the MHLC scales: a condition-specific measure of locus of control. Journal of Personality Assessment, 63(3), 534-553.

DOI:10.1207/s15327752jpa6303_10

West, R. (1991). Computing for Psychologists. Chur, Switzerland: Harwood.

Worsley, K., Whitehead, K., Kandler, R., Reuber, M. (2011). Illness perceptions of health care workers in relation to epileptic and psychogenic nonepileptic seizures. DOI:

https://doi.org/10.1016/i.yebeh.2011.01.029 